\title{
A Packet Priority Approach to Mitigate Starvation in Wireless Mesh Network with Multimedia Traffic
}

\author{
Balamuralikrishna Potti \\ Associate Professor \\ Dept. of ECE, SMITW \\ Thummalapalem, Guntur \\ $\mathrm{AP}$, India
}

\author{
M.V.Subramanyam \\ Principal \& Professor \\ Santhi Ram Engg. College \\ Nandyal, AP, India
}

\author{
K.Satya Prasad \\ Professor, Dept. of ECE \\ UCE, JNTUK \\ Kakinada, AP, India
}

\begin{abstract}
Wireless Mesh Networks (WMN) faces the inherent problem of increased end to end delay over a multiple hop wireless link. The delay becomes more obvious as the number of hops count between the source and destination increases. Experimental results have shown two hop neighbours face starvation when there are one hop nodes within the same gateway. In this paper, it is proposed to study the effect of starvation in WMN for multimedia traffic which are governed by the end to end delay and jitter for QOS. A novel packet priority technique, Dynamic Weighted Round Robin (DWRR) is proposed to reduce the effect of starvation for multi hop nodes. The proposed technique is implemented and compared with network without packet priority techniques. Results show that the proposed technique can be used to reduce starvation in WMN.
\end{abstract}

\section{General Terms}

Wireless Mesh Network (WMN), End to end delay, Jitter

\section{Keywords}

Multimedia traffic, Packet priority, Starvation

\section{INTRODUCTION}

Based on ad-hoc networks, Wireless Mesh Network (WMN) transfers data to and from an Access Point (AP) that is linked to the Internet by a wired/ wireless network. These AP do not need to be within reach of network nodes. AP centered nodes forward packets from distant nodes to the AP, and if there are many nodes in the network, far away nodes then shift data with the AP in one or two hops. Other advantages, in addition to mobility for WMN, include decentralized working, being cheap for initial infrastructure, reliable, scalable and providing more coverage [1]. Without taking recourse to WMN, a few APs set up can schedule medium usage schedules for various network users. Users can use various routes based on routing protocols. A WMN is also an excellent last-mile option for Wireless Broadband Access.

There are proactive and reactive routing protocols in ad hoc networks. The former have to maintain routes between node pairs always, while reactive routing protocols [2, 3] build/maintain routes based on demand. Studies reveal that reactive routing protocols perform better with regard to packet delivery ratio, incurring lower routing overhead specially during high mobility $[4,5]$. WMN being a special case of ad hoc networks, the communication to the external world is through APs and hence in multi hop WMN, the goal of nodes is to establish a two way communication with the AP. Every node issues route requests to neighbours. When they reach different APs, they send a route reply. The sending node accepts all replies deciding which route and AP to use based on various parameters. As data transfer in ad hoc networks is the same as this, existing ad hoc routing protocols like DSR [2] and AODV [3] can be used. But they assume some properties of ad hoc networks which do not hold good for WMN [6].

In ad hoc networks, most transfer could be among various network computers with network usage being spread over many routes. Unlike ad hoc networks, majority of data transfer is between nodes and few APs in WMNs. Also most ad hoc protocols choose a direct and shortest route to destinations. Some network paths are used more in comparison to others. So it leads to congestion when such protocols are used in WMN. Some APs are used more and others less. This can lead to busy nodes in some routes, with others being used rarely. Many overloaded route nodes could also lead to high collision rates, packet drops in queue and delays in the queue waiting. A connected problem is bandwidth wastage. Thus, there is increasing demand for efficient congestion management routing protocols.

In WMN, various constraints like collisions, traffic level, and buffer occupancy and battery power should be considered as consideration of one constraint alone is not enough. This is due to a complex relationship between various constraints. Multi-constrained routing is a NP-complete issue without a polynomial solution requiring various heuristics and soft computing techniques to solve them [8]. The QoS requirements are more stringent in case of real time applications involving video and voice transmission. For proper functioning of such applications, the network must provide QoS in terms of throughput, delay, etc. The traditional 802.11 is not supported for QoS for real time applications. The 802.11e is enhanced with QoS factors. But most of the current WLAN infrastructure is 802.11 based and do not support 802.11e. Hence, there is a requirement for development of IEEE 802.11 based QoS mechanism. Similarly in real-time traffic, TCP based traffic and UDP based traffic can be mixed within a single service provider class. Most UDP transmitters are completely obvious to drops and never lower transmission rate because of dropping. When TCP flows are combined with UDP flows and if congestion occurs, TCP by its virtue will continually lower their transmission rates letting up their bandwidths to UDP flows. This phenomenon leads to TCP starvation and UDP dominance.

Starvation exhibits as a throughput distribution wherein only a couple of dominating flows obtain very high throughput. The remaining starving flows receive very low throughput. 
Habitually, in WMN the starvation happens in network bandwidth utilization to nodes closer to the gateway blocks the channel rather than giving an option to the far away nodes. In wireless links, the bandwidth is shared among neighboring nodes whereas wired links have devoted bandwidth. The flows not only use the bandwidth all along its path, but also contend for the neighbor's bandwidths path. Such inter-flow intrusion effects bandwidth starvation for certain nodes as they encounter busy channels [9]. To overcome it, a routing metric helps routing protocols decide paths that stabilize the traffic load all along the flow path, and also minimize the inter-flow interference forced in the whole neighboring locale.

Starvation is addressed mostly through the use of a scheduling algorithm with a priority queue. Priority based Packet forwarding has been obtainable in off-the shelf routers (for e.g., in popular brand models Cisco and Juniper Networks). These methods are to be controlled for supervision their networks (e.g., peer-to-peer). It can have a substantial effect on the applications front, on the measurement tool's output precision, and on the network troubleshooting procedures effectualness [11]. The users, developers and all other network administrators have no specific knowledge and no way to get hold of it.

Network administrators put into effect priority/link-sharing mean in a router by describing a traffic class (usually IP protocol and TCP/UDP port number) and connecting with it an exact queuing/scheduling mechanism. The most common mechanisms are as follows.

- Priority Queuing (PQ). This permits users to allocate randomly defined packet classes to queues with various priorities. Since queues are sent out priority-wise, this lets particular packet types to be sent before others.

- Proportional Share Scheduling (PSS). With PSS all the traffic class is assigned a weight. Bandwidth is owed to classes based on their relevant weights. There is no severe priority disparity among classes. These scheduling mechanisms are incorporated in many ways, e.g., Weighted Fair Queuing (WFQ) (Cisco routers), Weighted Round-Robin (WRR) (Custom Queuing).

- Policing. This confines the traffic class's highest rate. Traffic that goes beyond the parameters rate is plunged. The traffic class is not able to scrounge for idle bandwidth from others.

Packet forwarding prioritization (PFP) in routers is a familiar method on hand to network operators. PFP can affect the exactness of network measurements, and the network troubleshooting procedures [12].

In this paper, it is proposed to study the effect of starvation in WMN for multimedia traffic which are governed by the end to end delay and jitter for QOS. In the proposed study, the effect of packet priority and contention window is investigated for multimedia traffic.

\section{RELATED WORK}

Bali, et al., [13] portrayed a buffer management scheme facilitating two types of prioritized service disciplines so as to get better QoS through cell delay time for every traffic class and to minimize the cell loss rate of loss sensitive class in an ATM network. The presentation analysis and relative study of these analyzed buffer management scheme (ATM networks) to assign two various classes of traffic needs with dissimilar QoS. The random traffic's delay time (ARBSD priority scheme) and the RSD priority scheme are dependent on the control parameter $\mathrm{R}$. When $\mathrm{R}$ goes low, the mid line delay time of the loss aware traffic class diminishes at the span of mid line delay time of the delay aware traffic class for both schemes. When traffic is loss aware, the normal delay time of loss aware traffic class reduces more, but the normal delay time for delay aware class goes high gradually. So it is clear that employing the ARBSD priority scheme and the RSD priority scheme is not a beneficiary act. When mainly traffic is delay receptive, the typical delay time of loss aware traffic class reduces a lot at the stretch of normal delay time of the delay receptive traffic class. So, reducing the starvation problem in the static priority scheme, it is efficient to employ the ARBSD priority scheme and the RSD priority scheme.

Ramesh, et al., [14] presented an inference tool (end-to-end) approach for PPF incidental from the feedback through routers. The proposed method was evaluated through statistical analysis. The users find network policies through measurement of packet losses of various packet types. Additionally, every connected network operators were reviewed and take delivery of responses in which half of them accepts the inferences. It is unable to discover a number of priority paths put into practice via traffic policing. It was established that the method which cause delay dissimilarity over packet types such as sluggish processing path in the router and port-based load allocation are found. During the experiment, the loss-based method finds out various multi priority paths in the Internet. In detecting for a method with less query overhead than the loss-based method, packet reordering and delay are used as the inference metrics and they were not as good as loss in finding packet forwarding priorities.

Christinal, et al., [15] presented a hybrid inference method to handle with network characteristics Viz., shared congestion, packet forwarding priority, network tomography and assess every methodology based on packet loss rate. The proposed mechanism depends on the packet loss statistics. This acts as the end-user network analysis tool to recognize shared congestion, packet forwarding priority and network tomography. Simulation results shows the hybrid inference methods at end-user level will lend a hand to the users and network administrators in an efficient way to make out network characteristics which are confidential at the router level through different approaches.

Ronasi, et al., [16] proposed an algorithm called Fair Binary Exponential Backoff algorithm (FBEB) which is an upgradation of Binary Exponential Backoff algorithm (BEB). The binary exponential backoff algorithm is an extensively used backoff algorithm, which regulates the contention window size by calculating the traffic in the nodes (in its communication medium) by the total successive collisions occurring to the same packet. The FBEB algorithm states that a node which broadcast a packet successfully will not retune its contention window for the subsequent transmission; it has to hold for a time $\mathrm{T}$. The other nodes get access to the channel as the node controls the resetting. By scheming the node for retuning will make other nodes to process the channel. The time $\mathrm{T}$ relays on the prior contention in the network.

Mansoor, et al., [17] proposed an algorithm known Starvation Mitigation Algorithm (SMA) for minimizing starvation. The gateway will behave as a determinator for the network and transmits the message to every other node when the network bandwidth is used by any single node. Then the node that is accessing the bandwidth will lower its contention window so that remaining nodes will use the bandwidth effectively and starvation will be lowered. Simulation results exhibits that the 
given algorithm employed to minimize the starvation in a network and mesh nodes that have broadcasted data packets should not be allowed to broadcast the other data packets forcefully. Starvation is reduced due to delay in the transmission of the excessive packets. This outcome is accomplished by changing the contention window for communications in the IEEE 802.11 protocol. The total Throughput is up and Delays in the network are alleviated.

\section{METHODOLOGY}

\subsection{Proposed Dynamic Weighted Round Robin (DWRR) Algorithm}

Traditional algorithms include the Random, Round-Robin (RR), Weighted Round-Robin (WRR), Least Load and Fastest Response algorithms. In the proposed packet priority technique, the packet priority weights are determined during run time using the current QOS parameters when making distribution decisions. As a result, dynamic packet prioritization algorithms significantly improve QoS over static algorithms. It is assumed that non real-time traffic are TCP flows and real-time traffic are UDP flows.

The new dynamic WRR algorithm schedule the traffic flows based on the following rules:

Rule 1: If the non-real time traffic is less than $20 \%$ (where $20 \%$ is the threshold value) of the total traffic, normal RR packet priority technique is used for traffic scheduling.

Rule 2: If the non-real time traffic is greater than $20 \%$ and less than $40 \%$ of the total traffic, packet priority algorithm serves non-real time traffic that is greater than the threshold value along with real-time traffic based on RR technique.

Rule 3: If non-real time traffic is equal to or greater than $40 \%$ of the total traffic, packet priority algorithm serves non-real time traffic by varying weights dynamically, based on QoS parameters. To improve QoS, based on hypothesis, all onehop nodes increase the contention window (CW) by $20 \%$ of the set value and all the two-hop nodes decrease the contention window by $10 \%$.

The pseudo code for the proposed algorithm is as follows:

Set Threshold traffic $=20 \%$

If non-real time traffic $<20 \%$

Then packet scheduling technique $=$ RR Technique with packet priority

Else

If $20 \%<$ non-real time traffic $<40 \%$ real-time

Then packet scheduling technique $=\mathrm{RR}$ to serve non time traffic

traffic more than the threshold value along with real-

\section{Else} packet

Then packet scheduling technique $=$ DWRR with

$$
\text { Priority }
$$

\section{Endif}

\section{Endif}

If hop distance $>1$

$$
\text { Then set } \mathrm{CW}_{\text {new }}=0.8 * \mathrm{CW}_{\text {old }}
$$

Else

Set $\mathrm{CW}_{\text {new }}=1.1 * \mathrm{CW}_{\text {old }}$

Endif

\section{EXPERIMENTAL SETUP}

In WMNs due to multihop links, the data packets face end to end delay. Higher the hop count, end to end delay becomes more apparent. In WMN all the flows converge at the gateway, and the presence of one hop nodes result in starvation for the multihop nodes. Simulation is conducted to study the effect of packet priority for multimedia traffic flows along with random TCP flows. AODV is used as the routing protocol for the network. The proposed packet priority technique uses a Dynamic Weighted Round Robin (DWRR) for proportional share scheduling. The weights are dynamically adjusted based on the end to end delay of the multimedia traffic. As the end to end delay increases the priority of the multimedia traffic increases proportionately till the stable state of 250 microseconds is achieved. Table 1 tabulates the simulated Network specifications.

Table 1: Simulation Specifications

\begin{tabular}{|l|l|}
\hline Number of Nodes & 20 \\
\hline Number of Gateways & 1 \\
\hline Area & $4 \mathrm{~km} \mathrm{X} \mathrm{4} \mathrm{km}$ \\
\hline Traffic & Multimedia with Random TCP flows \\
\hline Routing Protocol & AODV \\
\hline Simulation Time & $180 \mathrm{sec}$ \\
\hline
\end{tabular}

Two scenarios were considered with the first scenario without the proposed DWRR and the second scenario with DWRR.

\section{RESULTS}

The simulation results are shown in the following figures. Figures $1-5$ give the simulation results for route discovery time, data dropped, retransmission attempts, retry threshold, and throughput respectively. Table 2 tabulates the same.

Table 2: Simulation results

\begin{tabular}{|l|l|l|}
\hline Performance metrics & Normal & Priority \\
\hline & Mean & Mean \\
\hline Route discovery time in seconds & 0.8908605 & 0.71924 \\
\hline Data dropped bits/sec & 90328.933 & 43410.9 \\
\hline No.of Retransmission attempts & 0.6037692 & 0.54836 \\
\hline Retry threshold exceeded bits/sec & 2363.7778 & 2434.98 \\
\hline Throughput bits/sec & 660542.84 & 665911 \\
\hline
\end{tabular}




\begin{tabular}{|l|l|l|}
\hline & Min & Min \\
\hline Route discovery time in second & 0.0044096 & 0.00544 \\
\hline Data dropped bits/sec & 0 & 0 \\
\hline No.of Retransmission attempts & 0.0661765 & 0 \\
\hline Retry threshold exceeded bits/sec & 0 & 0 \\
\hline Throughput bits/sec & 0 & 0 \\
\hline & Max & Max \\
\hline Route discovery time in second & 2.1502572 & 1.86909 \\
\hline Data dropped bits/sec & 622200 & 311564 \\
\hline No.of Retransmission attempts & 0.7430830 & 0.72247 \\
\hline Retry threshold exceeded bits/sec & 9946.6667 & 9946.67 \\
\hline Throughput bits/sec & 2546848.9 & 2959076 \\
\hline
\end{tabular}

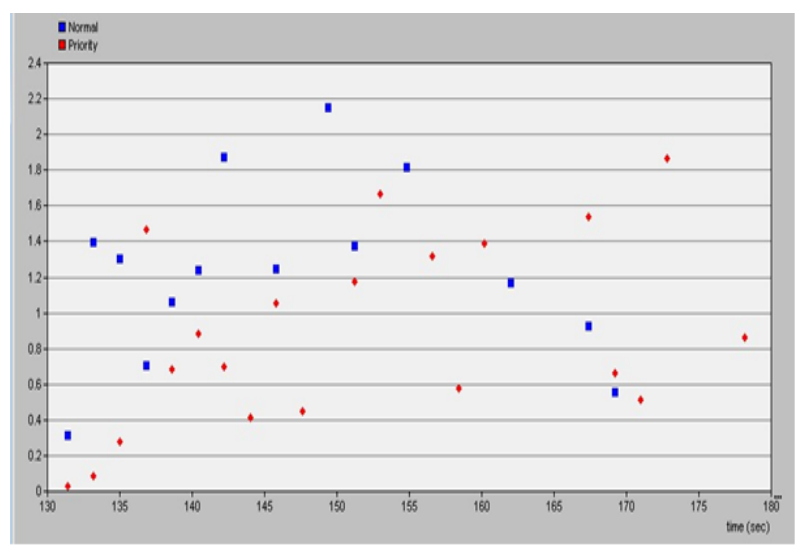

Figure 1: Route Discovery Time

It can be seen from Figure 1, that the route discovery time for multimedia data traffic when proposed packet priority is incorporated is lower than compared to routing without packet priority. Figure 2 shows the data dropped during transmission. The amount of data dropped drastically reduces when packet priority is applied.

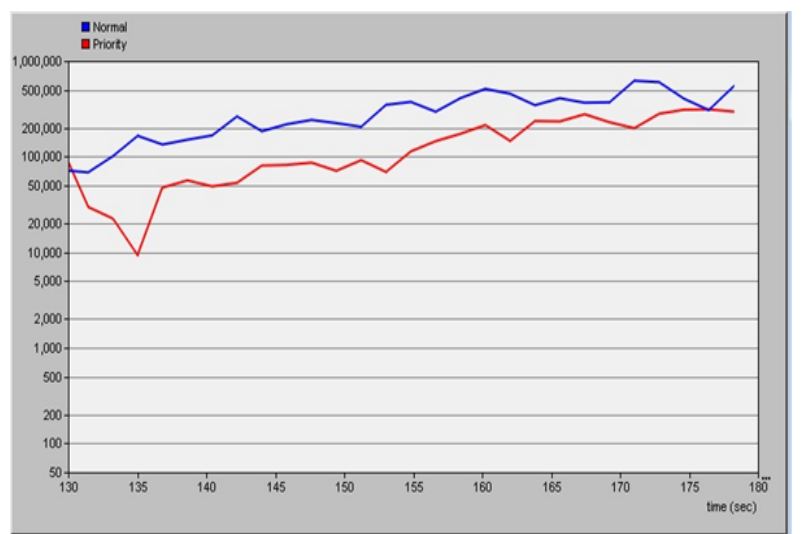

Figure 2: Data Dropped
Due to higher priority the data drop of multimedia packets reduce significantly by more than $50 \%$. Reduced drop in multimedia packets will reduce jitter significantly.

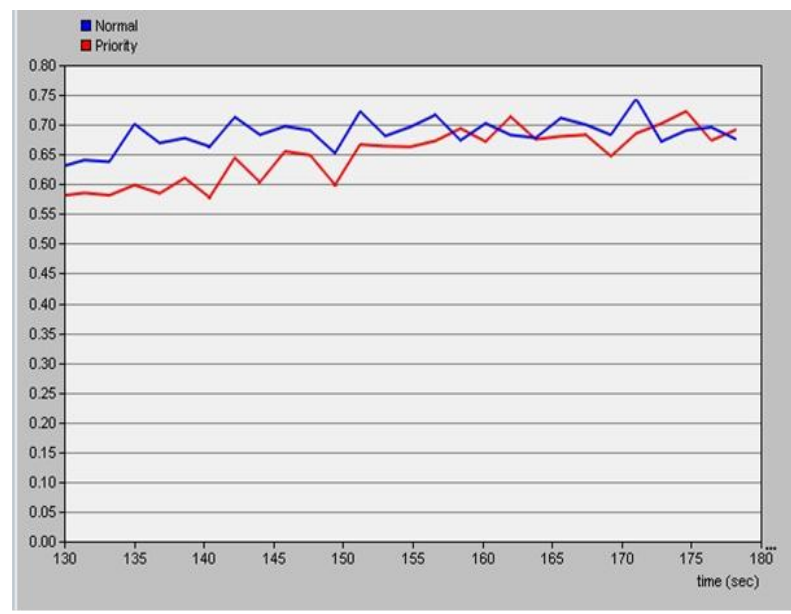

Figure 3: Number of Retransmission attempts

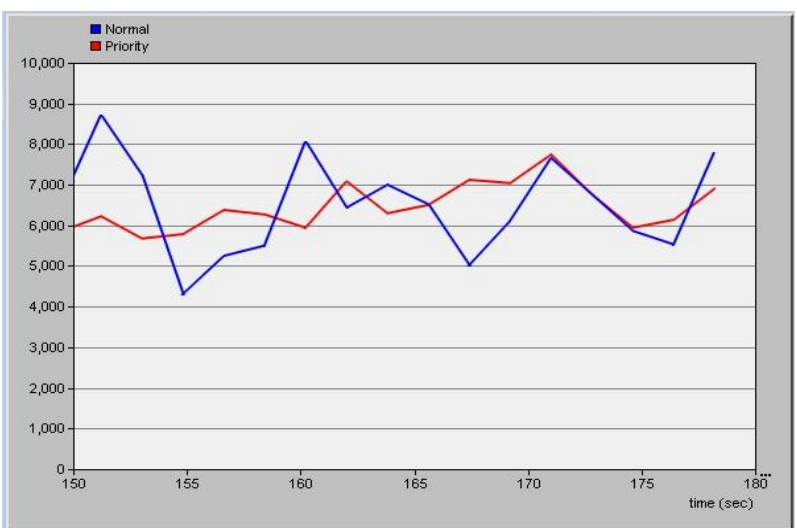

Figure 4: The Retry Threshold

The number of retransmission attempts and retry threshold shows a similar pattern in both scenarios. The retry threshold is more consistent when packet priority is considered.

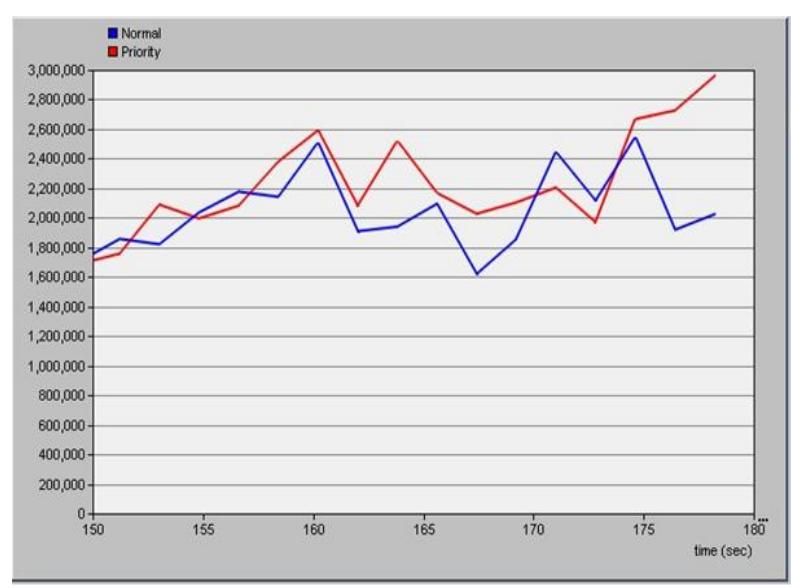

Figure 5: Throughput

From figure 5 it is seen that the throughput is better when packet priority is incorporated with AODV routing. It is evident from the simulation results that the packet priority incorporated in the routing for WMN improves the network's performance. 


\section{CONCLUSION}

In Wireless Mesh Networks (WMN) due to multihop links, the data packets face end to end delay. Higher the hop count, end to end delay becomes more apparent. In WMN all the flows converge at the gateway, and the presence of one hop nodes result in starvation for the multihop nodes. In this paper, the quality of service $(\mathrm{QoS})$ of WMN transmitting multimedia traffic is studied. Simulation is conducted to study the effect of packet priority for multimedia traffic. AODV is used as the routing protocol for the network. The simulation results illustrate the fact that the throughput is improved when packet priority is incorporated with AODV routing. It is also evident from the simulation results that the packet priority incorporated in the routing for WMN improves the network's performance. Further work needs to be done to improve the performance of WMN by incorporating dynamic contention window.

\section{REFERENCES}

[1] Mihail L. Sichitiu, "Wireless Mesh Networks: Opportunities and challenges", Proceedings of the Wireless World Congress, (Palo Alto, CA), May 2005

[2] David B. Johnson, David A. Maltz, and YihChunHu, "The Dynamic Source Routing protocol for mobile ad hoc networks (DSR)", July 2004, Available at http://www.ietf.org/internetdrafts/draftietfmanetdsr10.txt.

[3] C. Perkins, E. BeldingRoyer,and S. Das, "RFC 3561: Ad hoc On-Demand Distance Vector(AODV) Routing", July 2003, Available at http://www.ietf.org/ rfc/rfc3561.txt.

[4] E. Royer and C.K. Toh, "A review of current routing protocols for ad hoc wireless networks," IEEE Personal Communications, April 1999, pp. 4655.

[5] J. Broch, D. Maltz, D. Johnson, Y.C. Hu, and J. Jetcheva, "A performance comparison of multihop wireless ad hoc network routing protocols," in the Proceedings of the 4th ACM/IEEE Int. Conf. on Mobile Computing and Networking (MOBICOM) '98, Dallas, TX, USA, 1998, pp. 8597.

[6] Ann Lee and Paul A.S. Ward, "A Study of Routing Algorithms in Wireless Mesh Networks", Australian Telecommunication Networks and Applications Conference, December, 2004

[7] Xiaojing Tao, Thomas Kunz, David Falconer, "Traffic Balancing in wireless MESH networks", Proceedings of the International Conference on Wireless Networks, Communications, and Mobile Computing (WirelessCom 2005), Maui, USA, Jun 2005, Vol. 1, pp. 169174.

[8] T. Korkmaz and M. Kmnz, "Bandwidth delay constrained path selection under inaccurate slate information," IEEWACM TON, June 2003, Vol. II, Issue 3, pp. 384398.

[9] J. Shi, O. Gurewitz, V. Mancuso, J. Camp, and E. W. Knightly, "Measurement and Modeling of the Origins of Starvation in Congestion Controlled Mesh Networks", in Proceedings of IEEE INFOCOM, Phoenix, AZ, USA, Apr. 2008.

[10] Omer Gurewitz, Vincenzo Mancuso, Jingpu Shi, and Edward W. Knightly, "Measurement and Modeling of the Origins of Starvation of Congestion-Controlled Flows in Wireless Mesh Networks", IEEE/ACM Transactions On Networking, Vol. 17, No. 6, December 2009, pp: 1832-46.

[11] M. Aron, P. Druschel, andW. Zwaenepoel. "Cluster reserves: A Mechanism for resource management in cluster-based network servers" in Proceedings of ACM SIGMETRICS 2000, June 2000.

[12] G. Lu, Y. Chen, S. Birrer, F. E. Bustamante, C. Y. Cheung, and X. Li, "POPI: A User-level Tool for Inferring Router Packet Forwarding Priority," in Proc. IEEE INFOCOM, 2010.

[13] Vikram Bali, Rajkumar Singh Rathore, Amit Sirohi, "Performance Analysis of Priority Scheme in ATM Network", in 2010 International Journal of Computer Applications (0975 - 8887) Volume 1 - No. 13, PP: 2632 .

[14] D.V.Ramesh, D.Bujji Babu, S.Sreenivasulu, "Inference Tool Of Priority Based Packet Forwarding Across Network Via Feedback," International Journal Of Engineering Science \& Advanced Technology, Volume2, Issue-3, $747-753$.

[15] J.Benitha Christinal, V.Vivek, "Inferring Packet Forwarding Priority, Network Tomography and Congestion Control Based On Packet Probing In Network", International Journal on Computer Science and Engineering (IJCSE), Vol. 3 No. 1 Jan 2011, PP: 401-410.

[16] Ronasi, K., Gopalakrishnan, S., Wong, V.,"flow starvation mitigation for wireless mesh networks" Dept. of Electr. \& Comput. Eng., Univ. of British Columbia, Vancouver, 2009.

[17] Mansoor Rahaman, Ramesh Babu, Venkaiah Naidu, K. Rajasekhara Rao, "A New Starvation Mitigation Algorithm (SMA) for Wireless Mesh Networks", IJCST Vol. 3, Issue 1, Jan. - March 2012, pp:231-235 